\title{
Simultaneous AFM Nano-Patterning and Imaging for Photomask Repair
}

\author{
Aliasghar Keyvani ${ }^{a}$, Mehmet S. Tamer ${ }^{a}$, Maarten H. van Es, Hamed Sadeghian \\ Netherlands Organization for Applied Scientific research, TNO, Delft, Netherlands; \\ ${ }^{a}$ Also at: Department of Precision and Microsystem Engineering, Delft University of \\ Technology, Delft, Netherlands;
}

\begin{abstract}
In this paper we present a new AFM based nano-patterning technique that can be used for fast defect repairing of high resolution photomasks and possibly other high-speed nano-patterning applications. The proposed method works based on hammering the sample with tapping mode AFM followed by wet cleaning of the residuals. On the area where a specific pattern should be written, the tip-sample interaction force is tuned in a controlled manner by changing the excitation frequency of the cantilever without interrupting the imaging process. Using this method several patterns where transferred to different samples with imaging speed. While the pattern was transferred to the sample in each tracing scan line, the patterned sample was imaged in retracing scan line, thus the outcome was immediately visible during the experiment.
\end{abstract}

Keywords: Photomask Repair, AFM, Tip-sample interactions, Nano-Machining, Nano-Scribing.

\section{INTRODUCTION}

Scanning probe based nano-machining has many applications such as fabricating nano-wires, nano-dots, single electron transistors and many other devices for physics experiments. ${ }^{1-3}$ One of the applications that require high throughput nano-machining is repair of high photomasks for optical lithography.

Optical lithography is the main patterning technology for semiconductor industries. Thus, production, inspection and repair of high precision photomasks remain one of the challenges in high volume manufacturing. In this regards electron and focused ion beam microscopy based techniques are already in use for high precision masks repair, which each one has its own challenges and opportunities in achieving proper etching sensitivities, resolution and speed. ${ }^{4-6}$

Thanks to its nanoscale resolution, the atomic force microscopy (AFM) has already been suggested as one of the instruments for inspection and repair certain type of defects in 64 and 32nm node photomasks. However, there are still some challenges regarding the drift, positioning error and throughput of AFM based mask repair. ${ }^{7-9}$

When the particle defect is attached to the surface with a high adhesion so that wet cleaning fails to remove it, the AFM based cleaning is applied. The Photomask repair with AFM is done with a so called nano-machining process, followed by a wet cleaning process. ${ }^{8}$ The nano-machining process consist of multiple steps namely, locating and imaging the defect are in a gentle imaging conditions, deciding about the scan direction for removal process, and contact mode imaging of the particle. If the adhesion of the particle is low enough then the AFM tip pushes it away from its original location, so that it can be removed in the next wet cleaning process, otherwise the force set-point is gradually increased and the contact mode imaging is performed again, while focusing the scan area to the shape of the particle. Usually these steps which require multiple times scanning and scratching, with different cantilevers result in time consumption of about 2 hours per each defect.

An other challenge which the AFM based repair is facing is the positioning error and drift. it is very important to control the location of the defect during repair process, otherwise the repair process itself introduces new defects to the mask. New AFM based repair systems compensate for drift by forecasting its amount via so called edge detection technique.

a.keyvanijanbahan@tudelft.nl

Metrology, Inspection, and Process Control for Microlithography XXX, edited by Martha I. Sanchez, Vladimir A. Ukrainstev Proc. of SPIE Vol. 9778, 977818 · (c) 2016 SPIE · CCC code: 0277-786X/16/\$18 · doi: 10.1117/12.2219041 
In this paper we present a new nano-machining method based on the tapping mode AFM (TM-AFM) which can be used in photomask repair as well as other applications. The proposed method replaces the scratching process with hammering simultaneously with imaging. For this aim the tip-sample interactions are controlled via operation parameters of the TM-AFM so that the AFM can be quickly switched between imaging and hammering modes. Since the imaging and nano-machining are performed simultaneously the process can be performed much faster and drift control can be done much easier. Also there is no need to determine any scratching direction or use of multiple cantilevers. This paper is organized as follows: first we introduce the effects of operation parameters on tip-sample interactions in Section 2 via a theoretical and experimental results. In Section 3 we present the method which the nano-machining is performed together with preliminary results.

\section{TIP-SAMPLE INTERACTIONS IN AFM}

In tapping mode AFM the tip hits the surface in every tap, thus encounters both attractive van der Waals and repulsive contact forces in each cycle. But since the cantilever is highly frequency selective, it only gets affected by the zeroth and first frequency component of the tip sample interactions, namely the static amount and the first harmonic average. This fact makes it impossible to reconstruct the tip-sample interactions from the motion of cantilever, therefore the forces are not directly available via measurements. That is why determining the interactions between tip and the sample in tapping mode AFM has been a fundamental challenge for decades.

The damage in tapping mode AFM can be attributed to the Peak Repulsive Force (PRF) which is the maximum amount of the tip-sample interactions during each cycle. In the literature of AFM the PRF or tipsample forces are considered as a function of the amplitude and stiffness of the cantilever. However, the excitation frequency is actually by far the most important imaging parameter that affects the tip-sample forces. In this section we investigate the tip-sample interactions for different operation parameters (i.e., the excitation frequency and amplitude ratio) via a theoretical model and validate it with specifically designed experiments.

\subsection{Mathematical modeling}

In steady state conditions when the cantilever is moving harmonically, the motion of the cantilever can be well described with a one DOF mass spring model. Although, 1 DOF model is not always precise ${ }^{10}$ it is enough to describe the major effects of operation parameters on tip-sample interactions. Thus, the governing differential equation for tip can be written as:

Here, as the force model we consider the well-known DTM model, which consists of Hertzian repulsive and $\mathrm{vdW}$ attractive forces. Thus, the governing differential equation for tip can be written as:

$$
m \ddot{x}+c \dot{x}+k x=\frac{H R}{6\left(g_{0}-x\right)^{2}}-\frac{4}{3} E_{e f f} \sqrt{R} \delta^{3 / 2}+F_{0} \cos (\omega t)
$$

where $x$ represents the displacement of the tip, $k, c, m, R$ and $H$ are spring constant, damping ratio, effective mass, tip radius and Hamaker constant. $\delta=\left(a_{0}+x-g_{0}\right) H\left(a_{0}+x-g_{0}\right)$ is the indentation of the tip in sample for which, the Heaviside function $(H)$ is implemented to indicate that it is zero when tip is not in contact with sample and $a_{0}$ is the atomic separation distance. $E_{\text {eff }}$ is the effective stiffness of the surface that can be calculated as $\frac{1}{E_{e f f}}=\frac{1-\nu_{t}^{2}}{E_{t}}+\frac{1-\nu_{s}^{2}}{E_{s}} \cdot 11$

The Eq(1) can be solved with any nonlinear time integration method (e.g., Rung-Kutta) however it can take extreme amount of computational time to evaluate every single operation condition. In order to reduce the computational effort, we use a semi-analytic method similar to the method of $\mathrm{Hu}$ and Raman. ${ }^{12}$ To this aim, we first rewrite the equation in non-dimensional format, then transfer it to state space and rewrite the equations in terms of amplitude and phase (instead of displacement and velocity). Applying the periodic averaging method two integro-differential equation is achieved that can be solved semi-analytically with relatively simple computations.

To this aim we need to introduce the free air amplitude $\left(A_{i}\right)$, Amplitude ratio $\left(A_{r}\right)$, quality factor $(Q=$ $\left.\frac{\sqrt{k m}}{c}\right)$, frequency ratio $\left(\Omega=\frac{\omega}{\sqrt{\frac{k}{m}}}\right)$, non-dimensional dither force $\left(f_{0}=\frac{F_{0}}{k A_{i} A_{r}}\right)$, non-dimensional tip-sample force $\left(f_{t s}=\frac{F_{t s}}{k A_{i} A_{r}}\right)$, and time scale for slow dynamics $(\tau=\omega t)$. 
Applying the aforementioned steps to $\mathrm{Eq}(1)$, the $\mathrm{Eq}(2)$ represents the evolution of the amplitude and phase respectively.

$$
\begin{gathered}
\dot{A}=-\frac{1}{2 \Omega^{2}}\left[\frac{\sqrt{\left(1-\Omega^{2}\right)^{2}+\frac{\Omega^{2}}{Q^{2}}}}{A_{r}} \sin (\varphi)+\frac{A \Omega}{Q}+\frac{1}{\pi} \int_{a}^{2 \pi+a} f_{t s} \sin (\theta) d \theta\right], \\
A \dot{\varphi}=-\frac{1}{2 \Omega^{2}}\left[\frac{\sqrt{\left(1-\Omega^{2}\right)^{2}+\frac{\Omega^{2}}{Q^{2}}}}{A_{r}} \cos (\varphi)+\left(\Omega^{2}-1\right) A+\frac{1}{\pi} \int_{a}^{2 \pi+a} f_{t s} \cos (\theta) d \theta\right] .
\end{gathered}
$$

Where the amplitude $(A)$ is a non-dimensional variable that is related to the real physical amplitude as $A_{\text {real }}=$ $A A_{i} A_{r}$ and in engaged situation its amount is approximately one. considering the non-dimensional force model as:

$$
f_{t s}=\frac{\alpha}{(1-x)_{H \gamma}^{2}}-\beta(\gamma+x-1)_{H_{0}}^{\frac{3}{2}},
$$

where $\alpha=\frac{H R}{6 k Z^{3}}$ is the vdW gain, $\beta=\frac{4 E_{e f f} \sqrt{R Z}}{3 k}$ is Hertz gain, and $\gamma=\frac{a_{0}}{A_{i} A_{r}}$.

Neglecting the non-conservative interactions between the tip and the sample results in $\int_{0}^{2 \pi} f_{t s} \sin (\theta) d \theta=0$, and considering the force model as presented in $\mathrm{Eq}(3)$, we can write:

$$
\int_{0}^{2 \pi} f_{t s} \cos (\theta) d \theta=\alpha I_{1}(A, \gamma)-\beta I_{2}(A, \gamma)
$$

Since the range of non-dimensional amplitude in engaged situation is roughly known $(A \approx 1)$, the integrals $I_{1}$ and $I_{2}$ can be calculated separately for different amounts of $A$ and $\gamma$ and fitted to an algebraic function as following:

$$
\begin{gathered}
I_{1}=\int_{0}^{2 \pi} \frac{\cos (\theta)}{(1-A \cos (\theta))_{H \gamma}^{2}} d \theta \\
I_{2}=\int_{0}^{2 \pi} \cos (\theta)(\gamma+A \cos (\theta)-1)_{H 0}^{\frac{3}{2}} d \theta
\end{gathered}
$$

Finally the integro-differential equations $\mathrm{Eq}(2)$ can be reformulated as a set of nonlinear ordinary differential equations. Thus, for steady-state situations where $\dot{A}=\dot{\varphi}=0$, the tip-sample interactions for any amplitude ratio and frequency ratio can be calculated as the following set of nonlinear algebraic equations:

$$
\begin{gathered}
-\frac{1}{\pi} \zeta A I_{3}+\frac{A \Omega}{Q}+\sqrt{\left(1-\Omega^{2}\right)^{2}+\frac{\Omega^{2}}{Q^{2}}} \frac{\sin (\varphi)}{A_{r}}=0 \\
\frac{1}{\pi}\left(\alpha I_{1}-\beta I_{2}\right)+\left(\Omega^{2}-1\right) A+\sqrt{\left(1-\Omega^{2}\right)^{2}+\frac{\Omega^{2}}{Q^{2}} \frac{\cos (\varphi)}{A_{r}}}=0
\end{gathered}
$$

Solving the $\mathrm{Eq}(6)$ for the phase and amplitude, it is streght forward to find the peak repulsive forces from the force model in $\mathrm{Eq}(3)$. The results of the presented theoretical analysis together with an experimental verification are presented in Fig. 1.

\section{2 experimental measurement}

In order to experimentally measure the tip-sample interactions in TM-AFM, and investigate the effects of operation parameters on tip-sample interactions, we adopt the method presented by Tamer et al. ${ }^{13}$ In this method a secondary AFM cantilever with a separate optical beam deflection (OBD) sensor is used as a force sensor. The force sensing cantilever has a much higher resonance frequency and spring constant than the tapping cantilever. Thus it behaves as a rigid sample for the tapping cantilever yet, it is able to capture most of the frequency content of the tip-sample forces in its static band. Thanks to the high sensitivity of the OBD of the sensing cantilever the minute deflection is sensed, filtered and calibrated to reproduce the tip-sample interaction forces. Fig. 2 shows the force sensing setup which is used in this experiment. More details about this experiment are presented in. ${ }^{13}$ The measured and calculated peak repulsive forces are compared in Fig. 1 in terms of excitation frequency and amplitude ratio. Note that the results in Fig. 1 are calculated/measured considering a constant free air amplitude. Both the theoretical and experimental results suggest that above-resonance excitation increases the peak repulsive forces. In the next section, we shall use this property to introduce the nano-machining technique. 
a) Theoretical

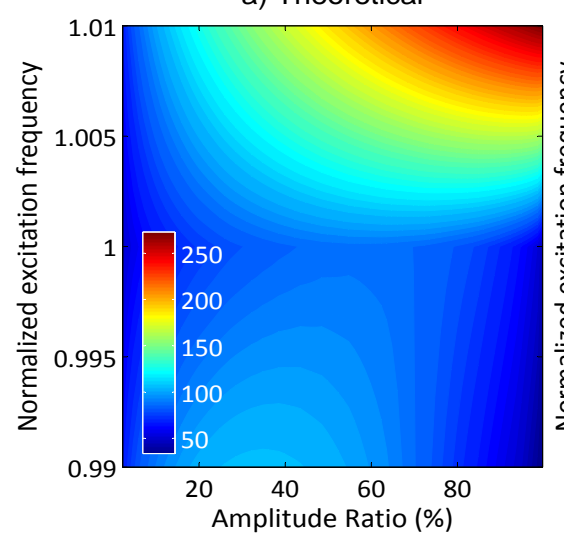

b) Experimental

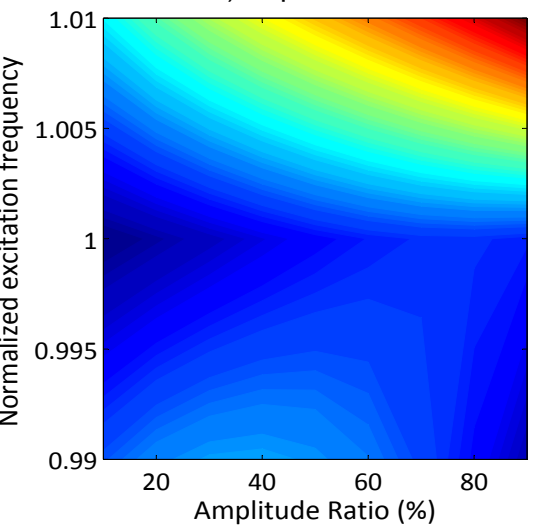

Figure 1. Normalized peak repulsive forces versus normalized excitation frequency (vertical) and amplitude set-point (horizontal). a) theoretical results according to Section 2.1, b) experimental results according to Section 2.2.

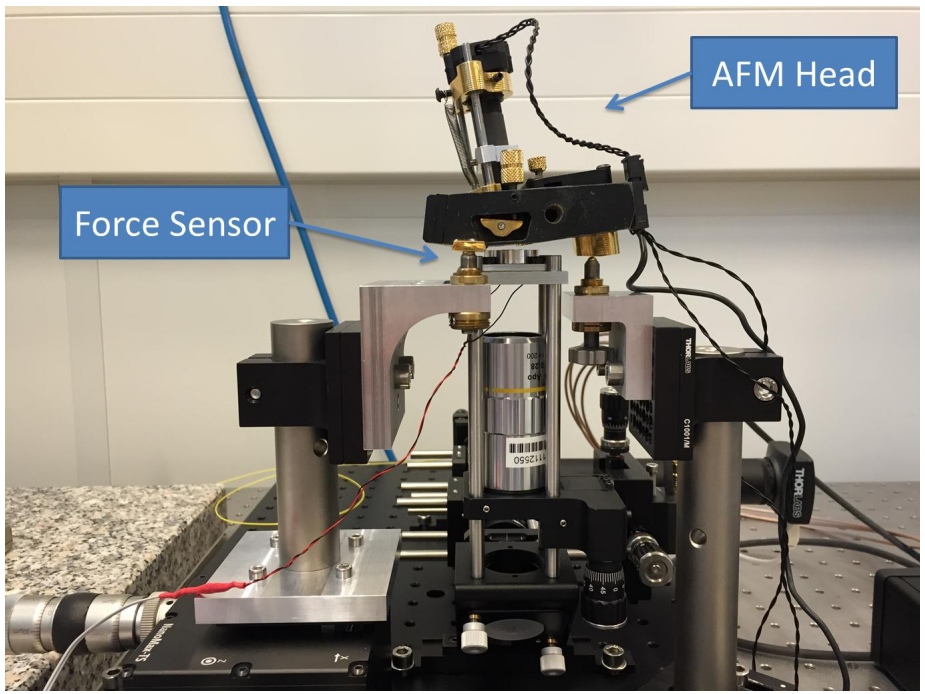

Figure 2. The force measurement setup for TM-AFM: the cantilever is tapping on a force sensor instead of the sample surface. 


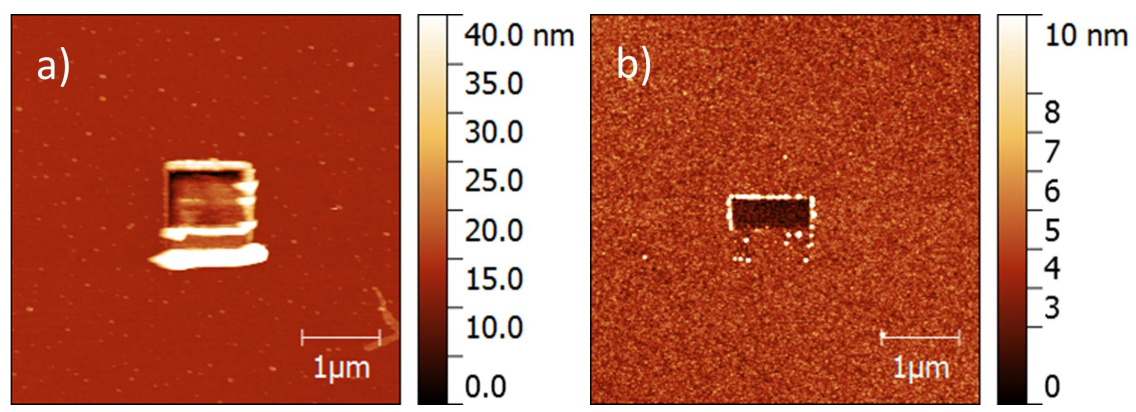

Figure 3. Nano-machining example by changing the excitation frequency using a diamond tip. a) HDMS coated on silicon b) silicon dioxide

\section{METHOD AND EXPERIMENTAL DEMONSTRATION}

Main goal of this paper is to introduce a nano-machining technique which can be used simultaneously with imaging by increasing the tip-sample interactions. The tip-sample forces are mainly attributed to the amplitude and stiffness of the cantilever which are practically very difficult to change (if not impossible) without interrupting the image. However, As shown in Fig. 1 the excitation frequency is actually by far the most important imaging parameter that affects the tip-sample forces. Provided that the free-air and set-point amplitudes are kept constant (i.e., staying in a vertical line in Fig. 1), one can increase and decrease the forces by changing the excitation frequency without interrupting the image.

The same dither voltage with two different frequency (one below and one above the resonance frequency of the cantilever) gives same free air amplitude and amplitude ratio (the later is because of closed loop control). However, these two situations give different peak repulsive force, specially for high amplitude ratios.

If the damage threshold of the material is between these two forces then one of the situations would be damaging and the other one would be non-damaging. In order to demonstrate the proof of concept for nanomachining, we first imaged a rectangular area on the sample with the so called writing parameters (high amplitude ratio and excitation frequency higher than the resonance of cantilever), then imaged a bigger area with its symmetric reading parameters (same free air amplitude and amplitude ratio but with lower excitation frequency). This experiment was repeated for different samples and with different free air amplitudes. The frequency offset, and free air amplitude of cantilever had to be calibrated for the depending on the tip and sample properties, and spring constant of cantilever to make sure that the writing configuration is damaging and reading configuration non-damaging.

Fig. 3 shows two examples of the experiment. One square written on a thin HDMS layer coated on silicon surface and the other one is a rectangle created on the surface of silicon dioxide. Both using a single crystal diamond tip in writing configuration, and immediately imaged with the same tip in reading configuration.

In order to demonstrate a semi-automatic nano-machining, we use an external computer programmable lockin amplifier (Zurich instrument) and a commercial AFM (Bruker Fastscan) with signal access module. The AFM was operated in contact mode however, deflection signal is isolated and sent to lock-in amplifier and the demodulated amplitude signal is provided back to the AFM as deflection error. In this manner the AFM controller keeps the amplitude constant, by trying to keep the deflection error constant in contact mode. This step was necessary because it was not possible to change the operation parameters via the AFM computer itself. The two different operation parameters (same voltage different frequency) are provided with Zurich Instruments Lock-in amplifier which was controlled with a separate computer program in Labview environment. The Labview code reads the location of the AFM head, compares it with a user provided image and decides about the excitation frequency. If the image color for accompanying pixel is black, the excitation frequency goes to writing mode and vice versa. In order to visualize the results immediately while doing the nano-machining, the aforementioned technique is only applied during the trace line, and retrace lines are only imaged with in reading mode. Fig. 4 is an example of nano-machining method where the TNO logo is printed on silicon dioxide sample with a diamond coated cantilever. 


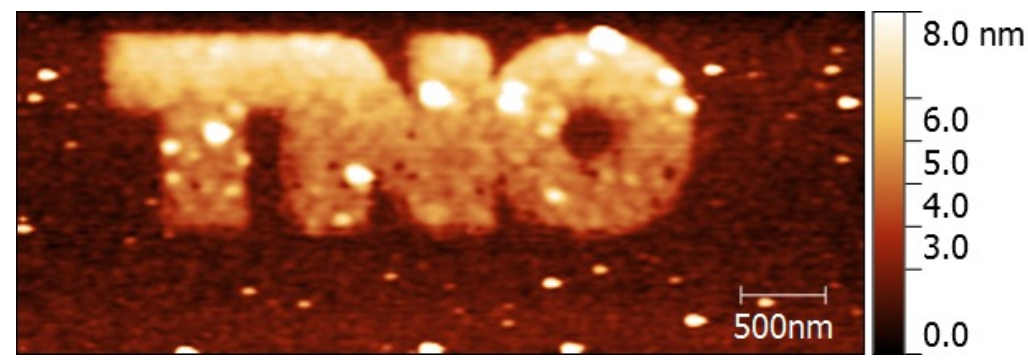

Figure 4. The TNO logo nano-patterned on silicon dioxide sample with diamond tip.

The cantilever which was used in this example had calibrated spring constant of $30.3 \mathrm{~N} / \mathrm{m}$ the quality factor of 300 and fundamental resonance frequency of $199 \mathrm{kHz}$, and was excited with free air amplitude of $85 \mathrm{~nm}$. The cantilever was engaged to the surface with a $90 \%$ amplitude ratio which assuming a nominal tip radius of $25 \mathrm{~nm}$ for reading frequency would give a peak repulsive force equal to $140 \mathrm{nN}$ while for the writing frequency the peak repulsive force would be $1.37 \mu N$.

The Nano-machining method which was suggested here offers a the possibility of the fast and automated photomask defect repair, nonetheless there are some challenges. First, similar to other machining methods, this method should have its own window of operation in terms of materials that can be used, maximum depth that can be achieved, resolution etc. However, these limits are not known for the authors yet. Secondly, a better hardware-software combination has to be developed to find the defect areas, compare the mask with the desired pattern, identify the defect type, and perform the repairing process. For the time being, only diamond coated tips was used in the experiments without any consideration about the sharpness or lifetime of the tips which also needs to be investigated.

\section{CONCLUSION}

In this paper the proof of principle for a new AFM based nano-machining technique was been presented. By investigating the tip-sample interactions in tapping mode AFM it was observed that the forces, not only scale with amplitude and spring constant, but also are very sensitive to the excitation frequency. Thus, the excitation frequency was adjusted to change the amount of peak repulsive forces to enable simultaneous reading and writing regimes for tapping mode AFM. The experimental results show that by changing only the excitation frequency it is possible to transfer a user specified pattern to the sample surface, and image it immediately in retrace lines. Seemingly the method can be useful in high speed mask repair with a resolution in the order of the tip-radius however a more general investigation on resolution, maximum speed and window of operation is still needed.

\section{ACKNOWLEDGMENT}

This works was supported by Netherlands Organization for Applied scientific Research, TNO, Early Research Program 3D Nanomanufacturing Instruments.

\section{REFERENCES}

[1] S Hu, a Hamidi, S Altmeyer, T Koster, B Spangenberg, and H Kurz. Fabrication of silicon and metal nanowires and dots using mechanical atomic force lithography. J. Vac. Sci. Technol. B, 16(5):2822-2824, 1998.

[2] Te-Hua Fang, Cheng-I Weng, and Jee-Gong Chang. Machining characterization of the nano-lithography process using atomic force microscopy. Nanotechnology, 11(3):181-187, 2000.

[3] Maria Ramiaczek-Krasowska, Adam Szyszka, Andrzej Stafiniak, Regina Paszkiewicz, Bogdan Paszkiewicz, and Marek Taczaa. Application of AFM technique for creation of patterns in nanoscale. Opt. Appl., 41(2):307-314, 2011. 
[4] M. Waiblinger, R. Jonckheere, Tristan Bret, D. Van den Heuvel, C. Baur, and G. Baralia. The door opener for EUV mask repair. 8441:84410F, 2012.

[5] Christian Ehrlich. Application data of the electron beam based photomask repair tool MeRiT MG. Proc. SPIE, pages 145-154, 2005.

[6] Fumio Aramaki, Tomokazu Kozakai, Osamu Matsuda, Osamu Takaoka, Yasuhiko Sugiyama, Hiroshi Oba, Kazuo Aita, and Anto Yasaka. Photomask Repair Technology by using Gas Field Ion Source. Bacus, 29(4):1-8, 2013.

[7] Tod Robinson, Roy White, Ron Bozak, Ken Roessler, Bernie Arruza, Dennis Hogle, Mike Archuletta, and David Lee. \&lt;title\&gt;New tools to enable photomask repair to the 32nm node\&lt;/title\&gt;. 7488:74880F$74880 \mathrm{~F}-12,2009$.

[8] M Dellagiovanna, H Yoshioka, H Miyashita, S Murai, T Nakaue, O Takaoka, a Uemoto, S Kikuchi, R Hagiwara, and S Benard. A semi-automated AFM photomask repair process for manufacturing application using SPR6300. Photomask Technol. 2007, 6730:673020, 2007.

[9] B LoBianco, R White, and T Nawrocki. Use of nanomachining for 100 nanometer mask repair. 19th Eur. Conf. Mask Technol. Integr. Circuits Microcompon., 5148(408):249-261, 2003.

[10] Robert Stark, Georg Schitter, Martin Stark, Reinhard Guckenberger, and Andreas Stemmer. State-space model of freely vibrating and surface-coupled cantilever dynamics in atomic force microscopy. Physical Review B, 69(8):085412, February 2004.

[11] Horacio V Guzman and Ricardo Garcia. Peak forces and lateral resolution in amplitude modulation force microscopy in liquid. Beilstein journal of nanotechnology, 4:852-9, January 2013.

[12] Shuiqing Hu and Arvind Raman. Analytical formulas and scaling laws for peak interaction forces in dynamic atomic force microscopy. Applied Physics Letters, 91(12):123106, 2007.

[13] Mehmet Tamer, Hamed Sadeghian, Hans Goosen, and Fred van Keulen. Quantitatively measuring nanomechanical, time-varying interaction forces in atomic force microscope imaging. Proc. of SPIE, 91(12):9778-89, 2016. 